\title{
1 FINE-SCALE POPULATION GENETIC STRUCTURE OF DENGUE MOSQUITO \\ 2 VECTOR, Aedes aegypti AND ITS ASSOCIATION TO LOCAL DENGUE 3 INCIDENCE
}

4 Thaddeus M. Carvajal ${ }^{1,2,3}$, Kohei Ogishi ${ }^{1}$, Sakiko Yaegeshi ${ }^{4}$, Lara Fides T. Hernandez ${ }^{1}$,

5 Katherine M. Viacrusis ${ }^{1}$ Howell T. Ho ${ }^{5}$, Divina M. Amalin ${ }^{2,3}$, and Kozo Watanabe ${ }^{1,2,3^{*}}$

$6{ }^{1}$ Department of Civil and Environmental Engineering - Ehime University, Matsuyama,

7 Japan

$8{ }^{2}$ Biology Department - De La Salle University, Taft Ave Manila, Philippines

$9 \quad{ }^{3}$ Biological Control Research Unit, Center for Natural Science and Environmental

10 Research - De La Salle University, Taft Ave Manila, Philippines

$11{ }^{4}$ Department of Civil and Environmental Engineering, University of Yamanashi, Kofu,

12 Japan

$13{ }^{5}$ Office of the Vice President of Academic Affairs, Trinity University of Asia, Quezon City, 14 Philippines

$15 *$ Corresponding author

16 Email: watanabe_kozo@cee.ehime-u.ac.jp (KW)

17 ABSTRACT

18 Dengue fever is an important arthropod-borne disease which is transmitted by the mosquito

19 vector, Aedes aegypti. Vector control programs rely heavily on targeting the mosquito 20 vector in order to stop the disease transmission cycle. Hence, the present study conducted a 21 fine-scale population genetics of Ae. aegypti in a highly urbanized and dengue endemic 
22 region in the Philippines. Furthermore, the study also explored the correlation of population

23 genetic indices to the local dengue incidence of the region. The genetic diversity and

24 population structure of Ae. aegypti populations were analyzed by genotyping 11

25 microsatellite loci from 526 adult mosquitoes sampled in 21 study areas in Metropolitan

26 Manila. Five genetic indices and its dengue incidence were then correlated using Pearson's

27 correlation. Results showed low genetic differentiation among mosquito populations

28 indicating high gene flow activity in the region. However, the study also revealed a

29 considerable number of inferred genetic clusters $(\mathrm{K}=5)$. The constructed UPGMA

30 dendrogram exhibited close proximity of genetically-similar Ae. aegypti mosquito

31 populations that extends in long distances suggesting passive dispersal ability of the

32 mosquito vector. Moreover, a positive and significant correlation was observed between

33 dengue incidence and inbreeding coefficient ( $F$ is $)(\mathrm{r}=0.52, \mathrm{p}=0.02)$. Overall, the study

34 showed that population genetic structuring can occur in a fine-scale area which consisted

35 notable clustering and extending patterns of genetically-similar mosquito populations. This

36 infers the potential migration ability of Ae. aegypti in different locations of the region

37 where specific vector control zones could be carried out to disrupt its dispersal ability. Also,

38 this is the first study that attempted to correlate genetic indices to dengue incidence that

39 could serve as a supplementary index in identifying high dengue risk areas in the future.

\section{AUTHOR SUMMARY}

Dengue disease puts billions of people worldwide at risk. To mitigate this risk, population genetic studies of its vector, Aedes aegypti, are being conducted. The information established from these studies can be utilized to reduce mosquito population and thereby, reduce the opportunity for dengue transmission. In this study, we used microsatellite markers to determine genetic structure and diversity followed by correlation analyses between genetic indices and dengue incidence. Results show a low genetic differentiation among mosquito populations in Metro Manila; it also indicates population genetic structuring in a fine-scale area. This suggest a pattern of migration activity of Ae. aegpyti which can be used to mitigate dengue transmission. Moreover, the study also explored in 
50 correlating genetic indices and local dengue incidence where it demonstrated significant

51 correlation with the inbreeding coefficient (Fis). Further investigation is needed on how

52 these genetic indices may be utilized in predicting and identifying high dengue risk areas in

53 endemic areas.

\section{INTRODUCTION}

Dengue disease is the most prevalent mosquito-borne viral infection in tropical and subtropical countries [1] with approximately 2.5 billion people worldwide at risk of contracting the disease [2]. Dengue virus is transmitted primarily to humans by the principal mosquito vector, Aedes aegypti. This mosquito species is considered to be the most efficient vector of arboviruses because of its highly adaptive nature to the urban environment [3]. Although a dengue vaccine is available [4], the World Health Organization [2] still recommends disease prevention and control towards the mosquito vector.

Molecular genotyping of the mosquito vector using microsatellites has provided useful insights towards the improvement of mosquito vector control strategies [5,6].For instance, revealing the gene flow pattern among Ae. aegypti populations can be interpreted as the mosquito vector's dispersal pattern [7,8]. Microsatellites is widely used as the standard molecular marker of choice in population genetic studies of Ae. aegpyti [9]. Due to the marker's high polymorphism, co-dominance, and broad genome distribution [10], it has been deemed suitable for differentiating both macro- and micro-geographic scale mosquito populations $[11,12,13]$.

Despite the many population genetic studies of Ae. aegypti using microsatellites worldwide, only a handful of studies have investigated the vector's genetic structure in a fine-scale area $[7,11,12,13,14,15,16]$. These fine-scale studies are defined as having sampling points within city boundaries, or villages with geographic distances of less than

$50 \mathrm{~km}$. For instance, spatial genetic differentiation across Ae. aegypti populations was evident in spatial scales within city boundaries $[7,11,12,16]$, among villages $[13,14]$ and 
77 along a street [15]. It has been claimed that genetic divergence in small spatial scales is common in Southeast Asia [11] and could also be attributed to the type of breeding sites [15]. Furthermore, multiple inferred clusters of genetic mosquito populations $(\mathrm{K}=3-9)$ were also detected within these fine-scale areas $[11,14,16]$. It was suggested that a single house or groups of closely situated houses may act as assembling units in forming these genetic clusters [11].

The information and patterns identified by the population genetic approach can be factored as part of the strategy in reducing the mosquito population, thereby, decreasing the opportunity of dengue transmission. Early fine-scale population genetic studies of Ae. aegypti $[7,8,11,15]$ had only demonstrated the degree or magnitude of genetic structuring while recent studies [12,16] concentrated on hypothesis testing such as the role urbanization in genetic divergence [14]. Although the results provided relative insights to our understanding of the mosquito vector, it lacks in demonstrating the clustering or distribution of genetically-similar mosquito populations which can reveal notable patterns for it application in vector control. For instance, in Yuunan Province of China, Ae. aegypti populations from border areas of the region are genetically-similar among each other and distinct from its two main cities indicating different invasion and colonization conduits [17]. The findings was presented simply in a dendrogram which can used in creating a sound basis in disrupting possible invasion of this mosquito vector from neighboring countries. In previous fine-scale population genetic studies, such graphical presentation was not illustrated but only described or portrayed in tabular records of pairwise genetic differences (e.g. $F_{\mathrm{ST}}$, Nei's genetic distance). Illustrating how geographical locations are geneticallysimilar or distinct may demonstrate the migration activity of Ae. aegypti and the extent of its spatial distribution patterns in fine-scale areas.

Surveillance of the immature or adult stages of Ae. aegypti has led to the conception of vector indices (e.g. Container, House, Breteau, Pupal or Adult indices) which can be utilized as a potential predictor of local dengue epidemiology [18]. However, frequent or consistent sampling of immature or adult stages of the mosquito vector has proven to be 
105 laborious and cost intensive [19]. Innovative and alternative avenues are now being explored in determining the population size of Ae. aegypti such as the development of non-

107 powered passive adult traps [20] and utilizing container-inhabiting mosquito simulation

108 approach [21,22]. One avenue that has not been explored is the application of genetic 109 indices that characterizes the mosquito vector population. For example, population genetics 110 can estimate the effective population size $(\mathrm{Ne})$ of the mosquito vector which is related to its 111 consensus size [23]. With several population genetic studies that have reported the 112 estimated population sizes of local Ae. aegypti populations [9,13,24], no study had 113 associated $\mathrm{Ne}$ or other genetic indices to the local dengue incidence. It was demonstrated 114 that sampling 25-30 individuals per local area is suitable for microsatellite-based 115 population genetic studies [25] where genetic analyses can determine mosquito population 116 size by conducting specific sampling episodes as compared to frequent or regular mosquito 117 collection surveillance. genetic structure of Ae. aegypti within a fine-scale area and, second, it explores the 121 provide a basis of creating new and innovative approaches in controlling this mosquito 122 vector in highly urbanized or endemic areas in the Philippines.

\section{METHODS}

Study area and Mosquito sampling

125 Metropolitan Manila, a highly urbanized area, is the National Capital Region (NCR)

126 of the Philippines with an area of $636 \mathrm{~km}^{2}$. It is located at the eastern shore of Manila Bay 127 in Southwestern Luzon (14 $50^{\prime}$ N Latitude, $121^{\circ}$ E Longitude), Philippines, Southeast Asia. 128 It is composed of 16 cities and 1 municipality with a total population of 12,877,253 [26]. 129 This area is the most urbanized region in the Philippines being the center of the national 130 government, economy, education and culture, country's leading business center, largest 131 manufacturing location and principal port for importation and exportation [27]. 
In this study, Metropolitan Manila was divided into 21 study areas (Figure 1 and Table S1). Initially, study areas are delineated per city which represents at least one per city. However, some cities comprised of either a large or small land sized area, thus designating more study areas. For example, the largest city, Quezon City, in the region was divided into 5 study areas based on its district boundaries. In order to standardize the land size covered by each study area, we merged two small neighboring cities, San Juan and Mandaluyong. The map layer of the administrative city boundaries of Metropolitan Manila was obtained from the Philippine Geographic Information System (GIS) Data Clearinghouse for further analysis [28].

Households on each study area were selected based on voluntary informed consent in collecting adult Ae. aegypti mosquitoes inside their premises. The number of households per study area ranged from $2-14$ with an average of 6 households and a total of 134 households. The maximum distance among households within study areas ranged from 1.39 $\mathrm{km}$ to $6.17 \mathrm{~km}$. Since the households are widely dispersed within each study area, we calculated the geographical midpoint (http://www.geomidpoint.com/) to assign a single georeferenced location for each study area in subsequent genetic analysis. The distance among study areas (midpoints) ranged from $2.85-39.66 \mathrm{~km}$.

Collection of Ae. aegypti mosquitoes was done by installing a commercially available mosquito UV Light Trap (Jocanima (C) in each household's outdoor premises for 3-5 days. Collected adult mosquito individuals were sorted, then identified accordingly based on the pictorial keys of Rueda et al. [29] and preserved in 99\% ethanol. Majority of population genetic studies in Ae. aegypti have either used only larval or reared larval to adult samples. For this reason, this could lead to a potential bias in estimating population genetic parameters due to the sampling of full sibling mosquito larvae [30]. This was evidence in the population genetic structure of amphibian larval samples that led to inaccurate estimate of differentiation among populations when compared to adult samples 
158 [31,32]. As such, this study targets adult Ae. aegypti samples than conventional egg, or 159 larval samples to prevent collecting mosquito full siblings. From a collection period of May 1602014 until January 2015, a total of 526 adult Ae. aegypti were collected and ranging from 16112 to 42 individuals per study area (Table S1).

\section{DNA Extraction and microsatellite genotyping}

The total genomic DNA of individual mosquito sample was extracted using the QIAGEN Blood and Tissue DNEasy Kit following the modified protocol of Crane [33]. We identified 11 microsatellites from Slotman et al. [34] and Chambers et al. [10] for genotyping and grouped them accordingly into four sets for multiplex PCR (Table S2). Each set consisted fluorescent labeled forward primers with different annealing temperatures during the amplification process. Generally, each set composed of $1.2 \mu \mathrm{L}$ of 10X buffer (TAKARA), $0.8 \mu \mathrm{L}$ of $25 \mathrm{mM} \mathrm{MgCl}_{2}, 1.6 \mu \mathrm{L}$ of $10 \mathrm{mM}$ of each dNTPs, $0.6 \mu \mathrm{L}$ of $10 \mu \mathrm{M}$ forward and reverse primers and $0.08 \mu \mathrm{L}$ of $5.0 \mathrm{U} / \mu \mathrm{L}$ of Taq DNA polymerase (TAKARA), $1.5 \mu \mathrm{l}$ of 10\% Dimethyl sulfoxide (DMSO) and $1 \mu \mathrm{l}$ of template DNA consisting a final volume of $10 \mu \mathrm{l}$. Thermocycle conditions are as follows: initial denaturation step of $94 \square \mathrm{C}$ for 5 minutes, denaturation step of $94 \square \mathrm{C}$ for 30 seconds, annealing step with temperature and duration (in seconds) of each primer set as indicated in Table S2, extension step of $72 \square \mathrm{C}$ for 30 seconds following 35 cycles and a final incubation step of $72 \square \mathrm{C}$ for 5 minutes. PCR amplicons were checked by electrophoresis in 3\% agarose gels stained with Midori Green (Nippon Genetics) and visualized under UV light using the Chemidoc XRS Chemiluminescent Gel Documentation Cabinet (BIO-RAD). Prior to fragment size analyses, multiplex PCR products were diluted in 1/15 water and then pooled together. $1 \mathrm{ul}$ of each diluted pool were added with $0.5 \mu \mathrm{l}$ of GS $500 \mathrm{Liz}$ Internal Size StandardTM (Applied Biosystems, USA) and HD formamide for a total volume of $20 \mu \mathrm{l}$. Fragment analysis of the amplified products were done using ABI 3500 Genetic Analyzer (Life Technologies) while genotyping is done using GeneMapper (Applied Biosystems). Microsatellite data were checked for error and the presence of null alleles with MICROCHECKER [35]. 


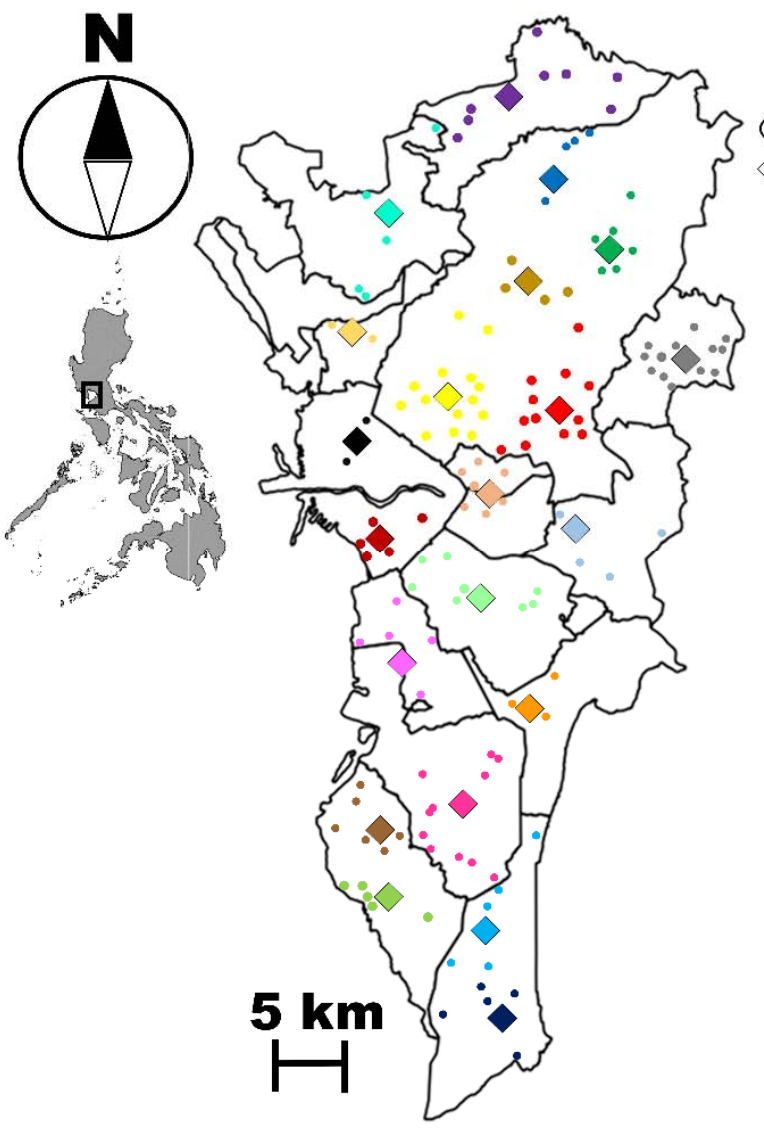

Households

Study Areas (City)

$\checkmark$ CAL-N (Caloocan City)

CAL-S (Caloocan City)

VAL (Valenzuela City)

QZC-1 (Quezon City)

QZC-2 (Quezon City)

QZC-3 (Quezon City)

QZC-4 (Quezon City)

QZC-5 (Quezon City)

MNL-1 (City of Manila)

MNL-2 (City of Manila)

MRK (Marikina City)

SJ-MAND (Cities of San Juan \& Mandaluyong)

PSG (Pasig City)

MKT (Makati City)

PSY (Pasay City)

TGG (Taguig City)

$\checkmark$ PRNQ (Parañaque City)

$\checkmark$ LSP-1 (Las Piñas City)

LSP-2 (Las Piñas City)

MTLP-1 (Mutinlupa City)

AMTLP-2 (Mutinlupa City)

\section{Figure 1. Geographic midpoints of Ae. aegypti study areas $(\diamond)$ with its corresponding} household sites ( $\odot$ ) in Metropolitan Manila. Details of each study area can be seen in Supplementary Table S1.

The exact Hardy-Weinberg equilibrium (HWE) test and estimations of the Linkage disequilibrium (LD) among all pairs of loci were conducted using GENEPOP v4.2.1 [36,37]. Significance levels for multiple testing were corrected using the Bonferroni procedure. The number of alleles, allelic richness and private alleles were calculated using HPRARE $[38,39]$. Observed heterozygosity $(\mathrm{Ho})$, expected heterozygosity $(\mathrm{He})$, inbreeding coefficients (Fis) were calculated using the Genetic Analysis in Excel (GenAlEx) version 
1966.3 [40]. To assess the magnitude of genetic differentiation among sites, pairwise $\mathrm{F}_{\mathrm{ST}}$ 197 values were calculated using Arlequin v3.5.1.3 [41] with 10,000 permutations. Pairwise 198 gene flow estimates $(\mathrm{Nm})$ among sites were manually calculated using the formula of 199 Slatkin and Barton [42] from the calculated pairwise F $_{\text {ST. A dendrogram was constructed }}$ 200 based on the pairwise $F_{S T}$ using the unweighted pair group with arithmetic mean (UPGMA) 201 in fastcluster package [43] and the optimal number of clusters were determined using the 202 cindex index in the NbClust package [44] from the R program [45].

\section{$\underline{\text { Genetic Structure }}$}

The number of genetic clusters $(\mathrm{K})$ was inferred using the Bayesian approach in the software STRUCTURE v2.3.2 [46]. The admixture model was utilized where its alpha value was allowed to vary, and independent allele frequencies was set at lambda equals to one. Twenty (20) independent runs were performed for each value of K $(1-15)$ with a burn-in phase of 200,000 iterations followed by 600,000 replications. Structure Harvester v0.6.93 [47] was used to determine the most likely number of clusters by calculating $\Delta \mathrm{K}$ [48]. Moreover, the software program CLUMPP v1.1.2 [49] was used to summarize the results from STRUCTURE and visualized using the program DISTRUCT v1.1 [50].

\section{Isolation by Distance and Spatial Autocorrelation}

Pairwise geographic distances $(\mathrm{km})$ among study areas and households were calculated using the Vincenty's formulae [51] on Microsoft Excel 2016. To test isolation by distance (IBD), pairwise $F_{S T}$ and geographic distance $(\mathrm{km})$ among study areas were examined using Mantel's test of correlation with 10,000 permutations. Spatial autocorrelation was performed using pairwise Nei's genetic distance among mosquito individuals and geographic distance $(\mathrm{km})$ among households with 10,000 permutations and Bootstrap replications. Results of the permutation were considered significant at the 5\% level. In this analysis, a correlogram was produced with 45 distance classes at $1 \mathrm{~km}$ interval. Both analyses yielded a correlation coefficient of the two data matrices ranging from -1 to 
$222+1$, with a test for a significant relationship by random permutation. All analyses were

223 performed using GenAlEx version 6.3 [40].

\section{Correlation Analysis between Genetic indices and Dengue incidence}

225 In order to calculate the dengue incidence of each study area, reported dengue cases

226 per village (baranggay) in 2014 were obtained from the National Epidemiology Center of

227 the Department of Health, Philippines while the population census per village were

228 acquired from the Philippine Statistics Authority agency (www.psa.gov.ph). Calculation of

229 dengue incidence was performed by dividing the number of cases to the total population

230 size for a given year multiplied by a factor of 1,000. Pearson's correlation coefficient was

231 calculated based from the computed dengue incidence and the selected population genetic

232 indices namely; Allelic richness, Private Allelic Richness, Observed heterozygosity,

233 Inbreeding coefficient and the effective population size. The correlation analysis was

234 performed using the stats package of the R program version 3.3.5 [45].

\section{RESULTS}

236 Genetic Diversity

237 We observed a total of 113 alleles across 11 microsatellite loci in 21 study areas 238 from Metropolitan Manila (Table S3). The number of alleles per loci ranged from 3 (F06) 239 to 19 (B07) with an average of 10.25 alleles per loci, suggesting that the chosen 240 microsatellites markers are highly polymorphic (Table S4). Null alleles were present in 4 241 loci (M313, AC4, AG7 and H08) and the null allele frequency ranges from $0.00-0.33$ in 242 all loci (Table S5). For the 231 tests of HWE of each locus per study area, 91 tests showed 243 statistically significant deviation $(\mathrm{p}<0.05)$ where 72 of these significant deviations 244 indicated $\mathrm{He}>\mathrm{Ho}$, suggesting heterozygosity deficits (Table S3). The LD test showed a 245 total of 119 of $1155(10.30 \%)$ pairs of loci with significant LD after Bonferroni corrections.

Table 2 shows the summary of the genetic diversity per study area. The mean 247 number of different alleles for all study areas ranged from 3.82 (TGG) to 6.36 (QZC-3) 
248 while the mean number of effective alleles for all study areas showed to be 2.74 (QZC-1) to

2493.51 (LSP-2). On the other hand, the mean allelic richness ranged from 3.24 (QZC-1) to

2503.85 (MRK) for all study areas while the proportion of private alleles ranged from 0.02

251 (LSP-2) to 0.23 (PSY). Overall, all study areas except for one (MKT) did not conform to

252 Hardy-Weinberg equilibrium expectations $(\mathrm{He}>\mathrm{Ho}$ ), indicating heterozygosity deficits

253 and the possibility of inbreeding within each study area. The effective median population

254 size $(\mathrm{Ne})$ across all study areas was calculated to be from 6.2 to 4,607 with two study areas

255 estimated as an infinite number.

256 Table 2. Dengue Incidence and Genetic Diversity among 21 Ae. aegypti populations 257 based on 11 Microsatellites in Metropolitan Manila, Philippines

\begin{tabular}{lcccccc}
$\begin{array}{c}\text { Study } \\
\text { Areas }\end{array}$ & $\begin{array}{c}\text { Dengue } \\
\text { Incidence }\end{array}$ & $\begin{array}{c}\text { Allelic } \\
\text { richness }\end{array}$ & $\begin{array}{c}\text { Private allelic } \\
\text { richness }\end{array}$ & $\begin{array}{c}\text { Observed } \\
\text { Heterozygosity }\end{array}$ & $\begin{array}{c}\text { Inbreeding } \\
\text { coefficient } \\
\text { (Fis) }\end{array}$ & $\begin{array}{c}\text { Median effective } \\
\text { population size } \\
\text { (Ne) })\end{array}$ \\
\hline CAL-N & 1.92 & 3.68 & 0.08 & 0.493 & 0.169 & 78.1 \\
CAL-S & 4.76 & 3.49 & 0.11 & 0.468 & 0.215 & 8.5 \\
VAL & 5.22 & 3.49 & 0.04 & 0.508 & 0.143 & 6.2 \\
QZC-1 & 0.25 & 3.24 & 0.10 & 0.470 & 0.111 & 116.1 \\
QZC-2 & 3.92 & 3.45 & 0.06 & 0.520 & 0.047 & 20.7 \\
QZC-3 & 3.26 & 3.44 & 0.03 & 0.525 & 0.071 & 57.7 \\
QZC-4 & 3.68 & 3.63 & 0.08 & 0.541 & 0.077 & 86.8 \\
QZC-5 & 4.45 & 3.37 & 0.05 & 0.500 & 0.117 & 58.2 \\
MNL-1 & 1.32 & 3.53 & 0.08 & 0.524 & 0.090 & 186.7 \\
MNL-2 & 3.70 & 3.55 & 0.03 & 0.468 & 0.132 & 386.8 \\
MRK & 3.64 & 3.85 & 0.07 & 0.511 & 0.112 & 4607.1 \\
SJ-MND & 4.08 & 3.29 & 0.03 & 0.496 & 0.191 & 38.1 \\
PSG & 2.81 & 3.56 & 0.08 & 0.524 & 0.099 & 31.7 \\
MKT & 2.02 & 3.59 & 0.06 & 0.558 & 0.036 & $\infty$ \\
PSY & 3.18 & 3.71 & 0.23 & 0.506 & 0.080 & 477.3 \\
TGG & 3.80 & 3.27 & 0.05 & 0.536 & 0.071 & 24.1 \\
PRNQ & 8.74 & 3.78 & 0.04 & 0.564 & 0.076 & 84.7 \\
LSP-1 & 8.37 & 3.65 & 0.12 & 0.536 & 0.104 & 38.4 \\
LSP-2 & 8.95 & 3.44 & 0.02 & 0.432 & 0.296 & $\infty$ \\
MTLP-1 & 8.44 & 3.79 & 0.13 & 0.438 & 0.240 & 79.4 \\
MTLP-2 & 7.51 & 3.51 & 0.03 & 0.449 & 0.240 & 94 \\
\hline
\end{tabular}


The overall $F_{\mathrm{ST}}$ was estimated to be 0.016 and pairwise $F_{\mathrm{ST}}$ values between study areas ranged from $-0.002-0.054$ (Table S6). With this, significant genetic differentiation

261 was demonstrated in 87 (out of 201, 41.4\%) pairwise values. Pairwise gene flow $(\mathrm{Nm})$ 262 estimates among study areas ranged from $3.404-290.448 .11$ pairwise gene flow estimates 263 were not calculated due to the estimated negative and zero $F_{\mathrm{ST}}$ values. The dendrogram 264 based on the pairwise $F_{\mathrm{ST}}$ values revealed the spatial pattern and distribution of genetically 265 similar study areas (Figure 2). Further analysis showed that the optimal number of cluster 266 groups is 6 where 4 indicated groups of genetically similar study areas. It is shown that 267 highly genetic-similar study areas are proximal to each other. This is exemplified by 268 genetic group 2 where mosquito populations in the south area except for LSP-2 are 269 genetically similar. There are also neighboring study areas that are genetically similar such 270 as in genetic groups 3 (VAL, CAL-S), 1 (QZC-2 and QZC-4) and 4 (PSG-MRK). 271 Furthermore, the pattern of the identified genetic groups extends in long distances as 272 demonstrated in genetic groups 1 and 4.

273 Mantel test between the pairwise genetic $\left(F_{\mathrm{ST}}\right)$ and geographic distances of all study 274 areas showed very low and non-significant correlation $\left(\mathrm{R}^{2}=0.01, \mathrm{p}=0.172\right)$, indicating no 275 isolation by distance. High genetic similarity among adult mosquitoes is limited up to $1 \mathrm{~km}$ 276 based on the spatial autocorrelation analysis (Figure S1). This suggests the limited dispersal 277 capability of Ae. aegypti.

STRUCTURE analysis found that the most likely number of genetically 279 differentiated groups is $\mathrm{K}=5$ (Figure S2). Figure 3 shows the distribution and proportion 280 of inferred genetic cluster assignment of each mosquito individuals per study area. It is 281 observed that either 4 or all inferred genetic clusters are present in each study area. 

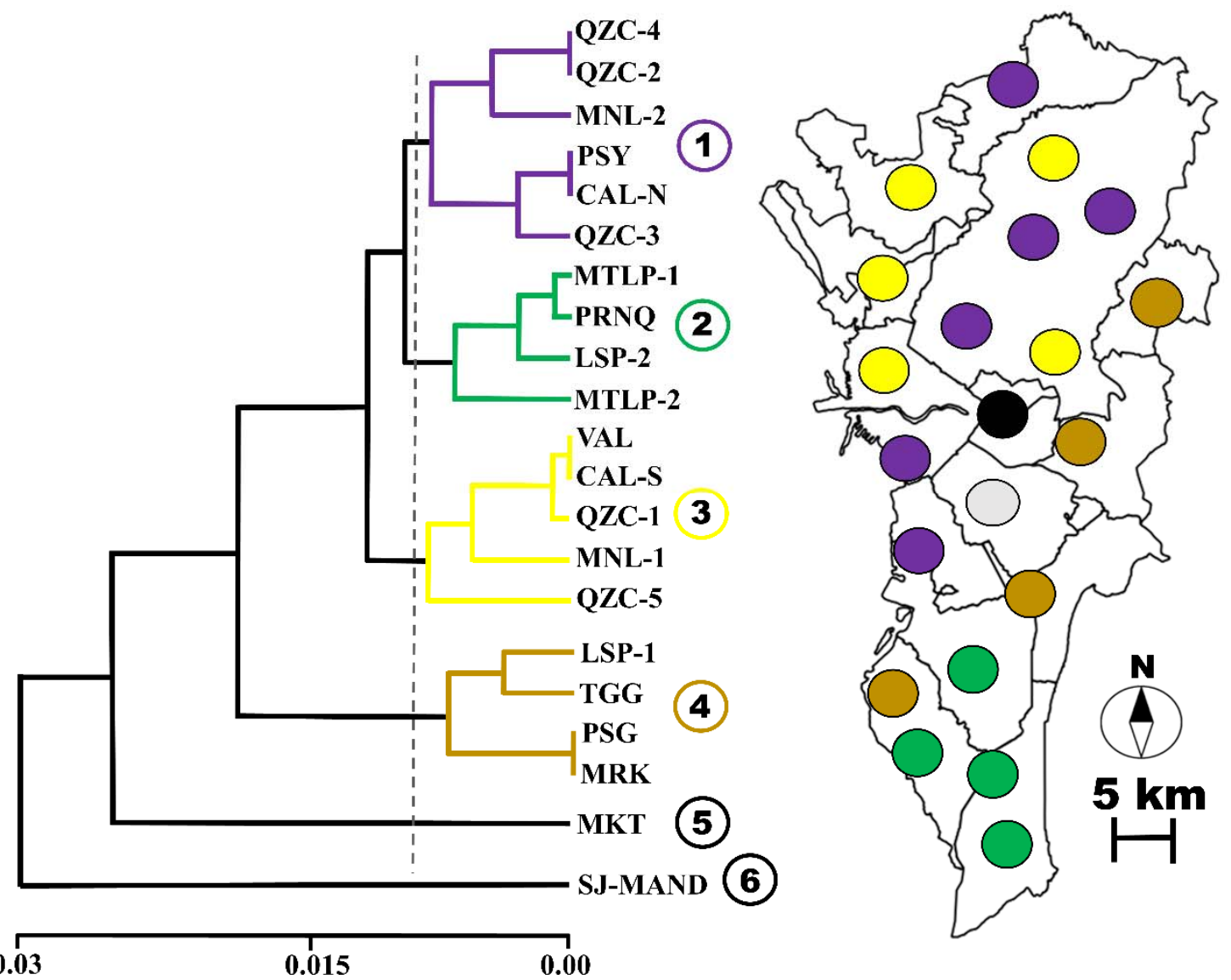

Figure 2. (a) Dendrogram showing the genetic relatedness of each study area based on

285 its pairwise $\boldsymbol{F}_{\text {ST }}$ estimates. Colored lines indicate the genetic groups. (b) Map showing

286 selected study areas in respect to their genetic group assignment. Colored circles

287 indicate the genetic group. 


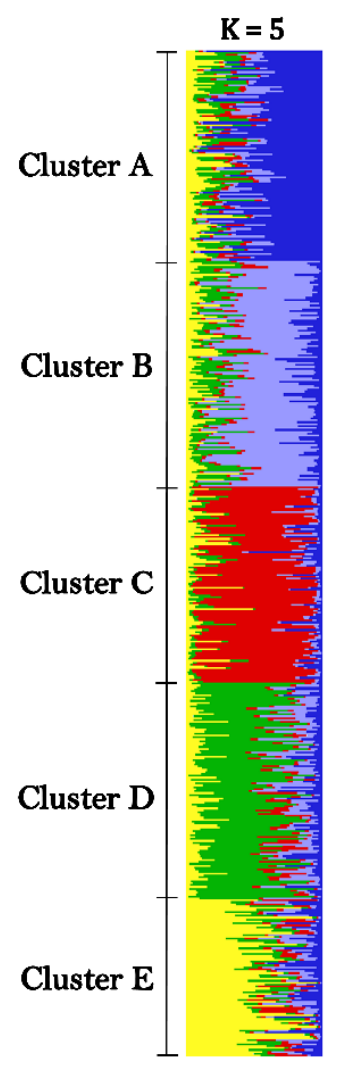

(a)

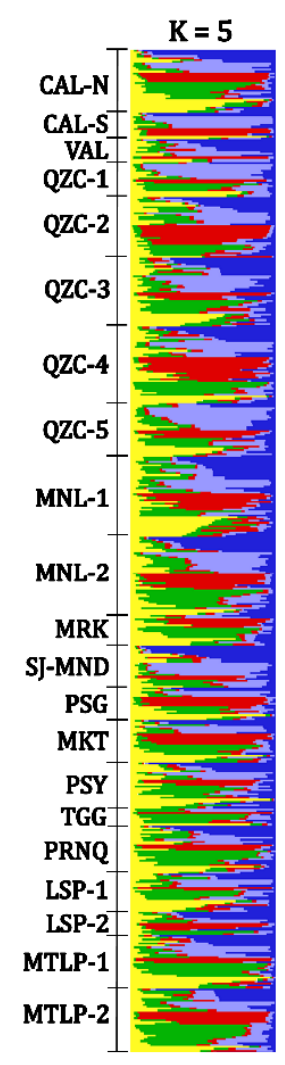

(b)

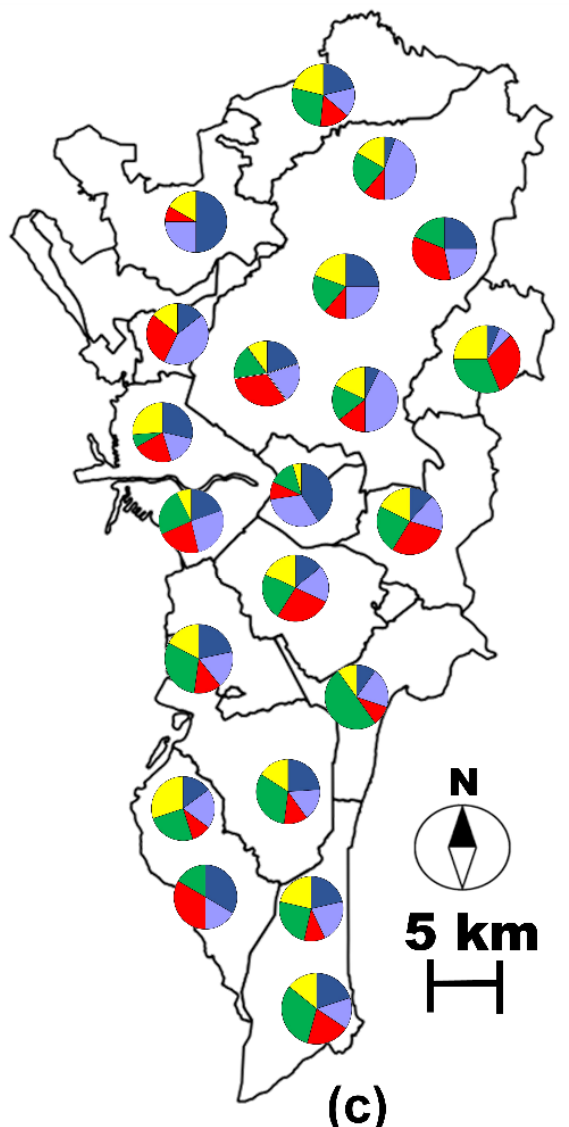

(c)
288

289

290

291

292

293

294

295

296

297

298

299

300

Figure 3. Bayesian analysis $(\mathrm{K}=5)$ of Ae aegypti populations in Metropolitan Manila. Bar plots represent the (a) genetic clusters and (b) study areas. Each individual is represented by a single horizontal line. Brackets are shown to separate genetic cluster or study areas. (c) Spatial map that shows the proportion of all genetic clusters to each study area. Colors represent the estimated individual proportion of cluster membership.

\section{Correlation between Population Genetic indices and Dengue incidence}

Five genetic indices were used to correlate with dengue incidence. It revealed that allelic richness $(r=0.30)$ and Fis $(r=0.52)$ showed a positive correlation to dengue incidence. Among these indices, it was only Fis that showed statistical significance (Figure 4). On the other hand, private allelic richness $(r=-0.14)$, observed heterozygosity $(r=-$ $0.26)$ and $\mathrm{Ne}(r=-0.10)$ demonstrated a negative correlation with no statistical significance. 


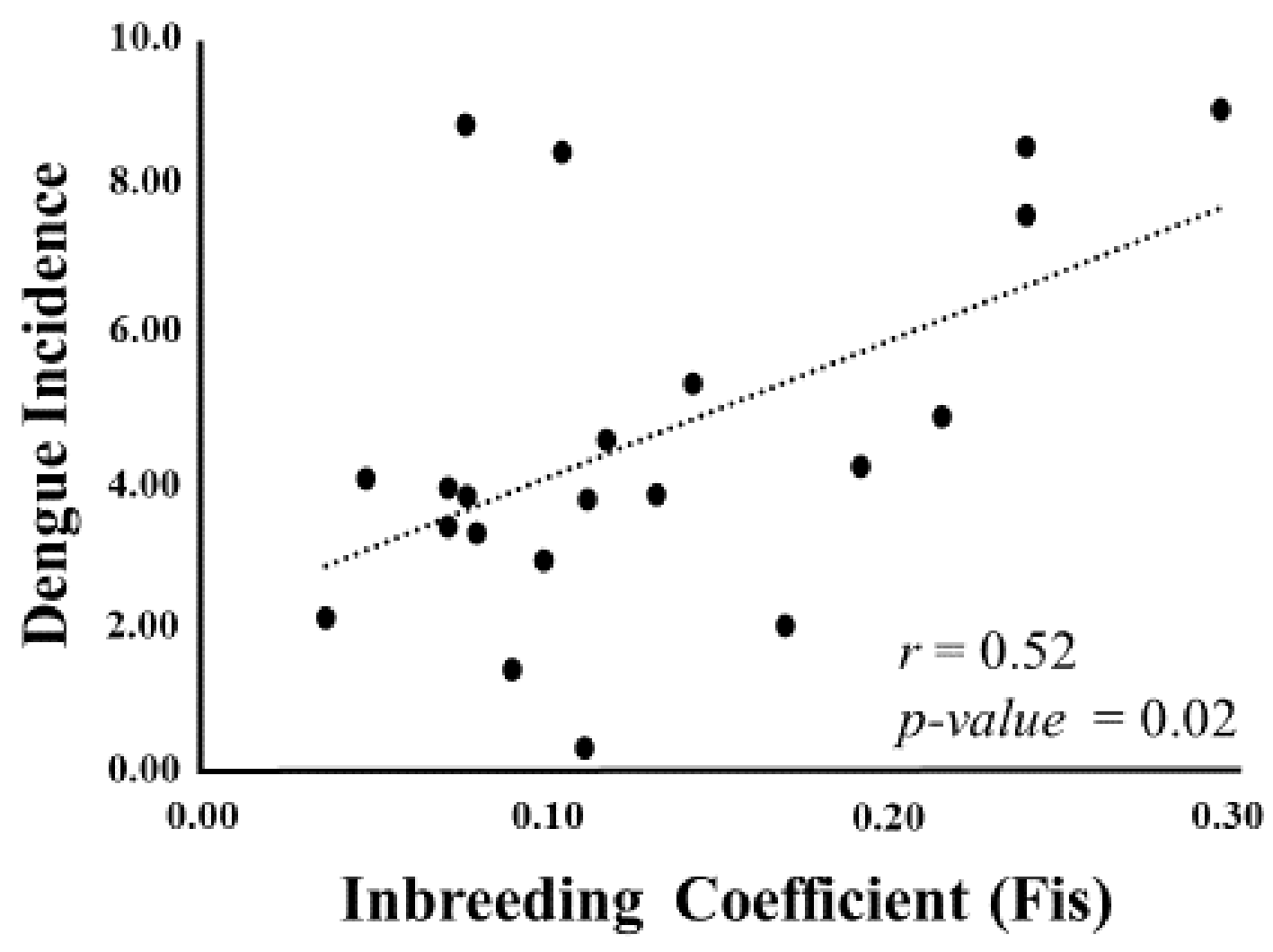

302 Figure 4. Correlation of Dengue Incidence and Inbreeding coefficient (Fis) among 21

303 study areas

DISCUSSION

$305 \quad$ Fine-Scale Genetic Structuring and Dispersal

306 Our study revealed low genetic differentiation among study areas $\left(F_{\mathrm{ST}}=0.006-\right.$ 307 0.054) which is similar from previous population genetic studies of Ae. aegypti that 308 consisted of a micro-geographic or fine-scale study area. In Sao Paulo, Brazil, for example, 309 the level of genetic differentiation ranged from 0.002 to 0.094 with a maximum distance 310 among collection sites of $30 \mathrm{~km}$ [14]. Cities in Southeast Asian countries also showed low 311 levels of genetic differentiation from $0.026-0.032$ with a spatial scale of $5-50 \mathrm{~km}$ [11]. 
312 The same was observed among villages in Thailand with geographical distances up to 27

$313 \mathrm{~km}$ showing an average genetic differentiation of 0.037 [13]. Our findings along with

314 previous studies suggest that mosquito populations within fine-scale areas may consist of

315 similar allele frequencies, thus, exhibit continuous and active exchange or sharing of alleles

316 among study areas. This is corroborated with the high gene flow estimates and the lack of a

317 detected signal of isolation by distance observed in the study.

Our constructed dendrogram showed that genetically similar Ae. aegypti mosquito populations are in close proximity with each other. This clustering pattern is highly 321 exemplified in the southern cities which comprises genetic group 2 (MTLP1, MTLP-2, 322 PRNQ, LSP-2) and the eastern part of the region (MRK, PSG and TGG). It demonstrates 323 that the dispersal of Ae. aegypti is limited which is also supported by our spatial 324 autocorrelation analysis. We can only infer such limited dispersal could be attributed by 325 landscapes in relation to the accessibility and location of each cities. For example, the 326 southern cities which comprises genetic group 2 (MTLP1, MTLP-2, PRNQ, LSP-2) can 327 only be accessed by one major highway while majority of the total land size of eastern 328 cities (MRK, PSG and TGG) are completely separated by a major highway and a river (e.g. 329 Marikina River). Therefore, access to these southern or eastern cities, may be difficult since 330 a few roads, highways, or bridges connect it from the rest of the region. For this reason, it 331 can potentially limit (but not isolate) the continuous migration and genetic exchange of 332 mosquito populations from other study areas in the entire region. This information provides 333 the potential migration or dispersal activity of Ae. aegypti that can be utilized in defining 334 specific vector control zones along landscape corridors (e.g. roads) within certain group of 335 cities.

What is also notable is that genetically-similar groups can extend in long distances such as observed in the northern to the central (genetic group 1) and the eastern to the southern parts (genetic group 4) of the region. If one overlays the major highways and road networks of Metropolitan Manila, it suggests the "passive" dispersal capability of Ae. 
341 aegypti by human-mediated transportation. It is believed that mosquito vectors occasionally

342 travel in long distances by taking advantage human-aided transportation routes via land, sea

343 or air [52,53,54] as Ae. aegypti eggs, larvae and adults have been found in commercial

344 trucks and ships through tire importation [55,56]. In addition, transportation zones such as

345 airports [57] and docks/ports [54,56] can be littered with larvae and pupae of the mosquito

346 vector, thus acting as the source population. Rapid urbanization (e.g. commercialization)

347 may also intensify this long distance migration or passive dispersal of Ae. aegypti by

348 promoting mosquito population admixture over distant areas [15,58].

Due to the high genetic similarity and migration activity observed among study areas, we expected a low number of inferred genetic clusters ( $\mathrm{K}=2$ at the most). However, it revealed a considerable number of genetic clusters $(\mathrm{K}=5)$. These findings are consistent 353 with previous population genetic studies with micro-geographic scales $[11,14,16]$ and local 354 studies in the Philippines [54,59]. Furthermore, these studies reported low genetic 355 differentiation but with substantial number of inferred genetic clusters $(K=3-9)$. The 356 substantial number of genetic clusters in fine-scale areas may be explained by two 357 hypotheses. The first hypothesis could be the result of divergence from a single ancestry 358 and, over time, produced multiple genetic clusters in this area. It is argued that a single or 359 closely-situated houses may act as a clustering unit in forming genetically structured 360 clusters $[11,52,60]$. This could be due to the limited flight performance of Ae. aegypti 361 where it prefers to stay within a small area of about 10 - 500 meters [61] for a stable 362 breeding site and availability of blood hosts [52]. As a result of rapid urbanization, these 363 distinct genetic mosquito groups may have migrated to distant locations through "passive" 364 dispersal, establishing colonies thereafter.

To some extent, our results support the first hypothesis where it revealed the limited 367 dispersal capability of Ae. aegypti in short distances (up to $1 \mathrm{~km}$ ) based from spatial 368 autocorrelation analysis and, in turn, may have generated the 5 inferred genetically 369 structured groups. Since Metropolitan Manila is considered highly urbanized with 
370 numerous transportation routes, it could have facilitated the passive dispersal of the

371 mosquito vector in distant locations based on the interpretation of high gene flow activity

372 among the study areas. However, limited dispersal cannot only be the single factor that can

373 explain the occurrence of multiple clusters. Without high mutation, the mosquito

374 population cannot diversify within a small spatial scale for a short evolutionary time. Hence,

375 the second hypothesis infers that it could be due to the immigration of mosquito

376 populations from neighboring regions or provinces of Metropolitan Manila, but a larger

377 spatial scale genetic data is needed to test this hypothesis.

378

379 Correlation of Genetic indices towards Dengue incidence

Among the genetic indices, we expected the estimated population size $(\mathrm{Ne})$ to show 382 a significant and positive correlation. However, it resulted in a non-significant and negative 383 relationship with dengue incidence. In our study, collecting adult mosquito samples was the 384 ideal choice to perform a better estimation of the effective population size $(\mathrm{Ne})$. Previous 385 population genetic studies conducted their sampling using either only larval samples or 386 larval samples reared to adult stages but majority of mosquito larvae do not become adults 387 in the natural setting [62]. Thus, collecting larvae to estimate the effective population size 388 of the adult may be deemed inappropriate.

One possible reason of detecting no correlation between $\mathrm{Ne}$ and dengue incidence 391 may be that the calculated $\mathrm{Ne}$ is either under- or over-estimated. Estimating the precise $\mathrm{Ne}$ 392 of natural populations can be difficult, especially if there is a large time interval between 393 sampling points or temporal disruptions such as migration or population replacement. It has 394 been demonstrated that lower $\mathrm{Ne}$ estimates are generated by the presence of temporal 395 disruptions while large intervals in sampling points generate higher $\mathrm{Ne}$ estimates [23]. 396 Nevertheless, the calculated $\mathrm{Ne}$ estimates of the study is consistent with those calculated by 397 Saarman et al. [23] from mosquito populations worldwide. But further and thorough 
398 investigation should be performed in the future to correctly estimate the $\mathrm{Ne}$ and its correlation with dengue incidence.

400

401

402

403

404

405

406

407

408

409

410

411

412

413

414

415

416

417

418

419

420

421

422

423

424

425

426

Notably, the correlation between dengue incidence and the inbreeding coefficient (Fis) revealed a positive and significant correlation $(\mathrm{r}=0.52, \mathrm{p}=0.02)$. One plausible mechanism may be due to the transovarial or vertical transmission of the dengue virus to its succeeding mosquito offspring. This viral transmission process has been demonstrated in field-collected Ae. aegypti mosquitoes until its F2 generation from Quezon City, Metropolitan Manila [63]. Earlier studies also revealed that the transovarial route can sustain the viral infection up to the $15^{\text {th }}$ generation [64]. Therefore, we infer that the inbreeding within the mosquito population may result in producing a substantial number of next generation mosquitoes carrying the dengue virus. This suggests that dengue virus infection is not only maintained by the human-vector cycle but also within mosquito generations. Because of such mechanism, it can possibly lead to an increased disease transmission within local areas. It should be known there are limitations in our interpretation of this correlation. First, no viral detection was done to each individual Ae. aegypti mosquitoes to ascertain their viral transmission capability. Performing this endeavor could provide the necessary credence that can strongly support such correlation. Secondly, it is unclear whether the dengue virus infection in the human population originated from the exact study area. It has been suggested that dengue infections in the human population especially in urbanized areas can be obtained from other locations such as public spaces, schools or workplaces rather than their place of residence $[65,66,67,68]$.

Nonetheless, this is the first attempt to directly link mosquito population genetic data to epidemiological data. Although several previous studies signified the application of population genetics towards vector control, they only reported the mosquito gene flow pattern and the association of mosquito genetic structure and its landscape [54,69,70]. Our findings may provide future implications in predicting or identifying high dengue risk areas where the genetic index (e.g. Fis) can be utilized as a supplementary index with 
427 conventional mosquito-based indices. But further research is needed to ascertain on how

428 these genetic indices can directly influence the local dengue epidemiology.

\section{REFERENCES}

1. Weaver SC, Reisen WK. Present and future arboviral threats. Antiviral research. 2010 Feb 1;85(2):328-45.

2. World Health Organization: Western Pacific Region (2012) Dengue: dengue in the Western Pacific region. Available: http://www.wpro.who.int/emerging_diseases/Dengue/en/index.html. Accessed June 2017

3. Arunachalam N, Tewari SC, Thenmozhi V, Rajendran R, Paramasivan R, Manavalan R, Ayanar K, Tyagi BK. Natural vertical transmission of dengue viruses by Aedes aegypti in Chennai, Tamil Nadu, India. Indian Journal of Medical Research. 2008 Apr 1;127(4):395.

4. Sanofi Pasteur. Sanofi Pasteur's Dengue Vaccine Approved in the Philippines. Available at: http://www.sanofipasteur.com/en/articles/sanofi-pasteur-dengue-vaccineapproved-in-thephilippines.aspx (2016). Accessed 26 Oct 2017

5. Hiragi C, Simões K, Martins E, Queiroz P, Lima L, Monnerat R. Genetic variability in Aedes aegypti (L.)(Diptera: Culicidae) populations using RAPD markers. Neotropical entomology. 2009 Aug;38(4):542-7.

6. Urdaneta-Marquez L, Failloux AB. Population genetic structure of Aedes aegypti, the principal vector of dengue viruses. Infection, Genetics and Evolution. 2011 Mar $1 ; 11(2): 253-61$.

7. Huber K, Le Loan L, Hoang TH, Tien TK, Rodhain F, Failloux AB. Temporal genetic variation in Aedes aegypti populations in ho chi Minh City (Vietnam). Heredity. 2002 Jul;89(1):7.

8. Ravel S, Monteny N, Olmos DV, Verdugo JE, Cuny G. A preliminary study of the population genetics of Aedes aegypti (Diptera: Culicidae) from Mexico using microsatellite and AFLP markers. Acta Tropica. 2001 Mar 30;78(3):241-50.

9. Rašić G, Filipović I, Weeks AR, Hoffmann AA. Genome-wide SNPs lead to strong signals of geographic structure and relatedness patterns in the major arbovirus vector, Aedes aegypti. BMC genomics. 2014 Dec;15(1):275.

10. Chambers EW, Meece JK, McGowan JA, Lovin DD, Hemme RR, Chadee DD, McAbee K, Brown SE, Knudson DL, Severson DW. Microsatellite isolation and linkage 
459 group identification in the yellow fever mosquito Aedes aegypti. Journal of Heredity. 2007 460 Apr 9;98(3):202-10.

461 11. Hlaing T, Tun $\square$ Lin W, Somboon P, Socheat D, Setha T, Min S, Thaung S, Anyaele 462 O, De Silva B, Chang MS, Prakash A. Spatial genetic structure of Aedes aegypti 463 mosquitoes in mainland Southeast Asia. Evolutionary applications. 2010 Jul 1;3(4):319-39.

464 12. Vidal PO, Suesdek L. Comparison of wing geometry data and genetic data for 465 assessing the population structure of Aedes aegypti. Infection, Genetics and Evolution. 4662012 Apr 1;12(3):591-6.

467 13. Olanratmanee P, Kittayapong P, Chansang C, Hoffmann AA, Weeks AR, Endersby 468 NM. Population genetic structure of Aedes (Stegomyia) aegypti (L.) at a micro-spatial scale 469 in Thailand: implications for a dengue suppression strategy. PLoS neglected tropical 470 diseases. 2013 Jan 10;7(1):e1913.

471 14. Wilke AB, Wilk-da-Silva R, Marrelli MT. Microgeographic population structuring 472 of Aedes aegypti (Diptera: Culicidae). PloS one. 2017 Sep 20;12(9):e0185150.

473 15. Paupy C, Chantha N, Huber K, Lecoz N, Reynes JM, Rodhain F, Failloux AB. 474 Influence of breeding sites features on genetic differentiation of Aedes aegypti populations 475 analyzed on a local scale in Phnom Penh Municipality of Cambodia. The American journal 476 of tropical medicine and hygiene. 2004 Jul 1;71(1):73-81

477 16. Louise C, Vidal PO, Suesdek L. Microevolution of Aedes aegypti. Plos one. 2015 478 Sep 11;10(9):e0137851.

479 17. Shi QM, Zhang HD, Wang G, Guo XX, Xing D, Dong YD, Xiao L, Gao J, Liu QM, 480 Sun AJ, Li CX. The genetic diversity and population structure of domestic Aedes aegypti 481 (Diptera: Culicidae) in Yunnan Province, southwestern China. Parasites \& vectors. 2017 482 Dec;10(1):292.

483 18. Bowman LR, Runge-Ranzinger S, McCall PJ. Assessing the relationship between 484 vector indices and dengue transmission: a systematic review of the evidence. PLoS 485 neglected tropical diseases. 2014 May 8;8(5):e2848.

486 19. KM, Versteirt V, Cull B, Kampen H, Fontenille D, Hendrickx G, Zeller H, Van 487 Bortel W, Schaffner F. An entomological review of invasive mosquitoes in Europe. 488 Bulletin of entomological research. 2015 Dec;105(6):637-63.

489 20. Ritchie SA, Cortis G, Paton C, Townsend M, Shroyer D, Zborowski P, Hall490 Mendelin S, Van Den Hurk AF. A simple non-powered passive trap for the collection of 491 mosquitoes for arbovirus surveillance. Journal of medical entomology. 2013 Jan $492 \quad 1 ; 50(1): 185-94$. 
493 21. Williams CR, Johnson PH, Ball TS, Ritchie SA. Productivity and population 494 density estimates of the dengue vector mosquito Aedes aegypti (Stegomyia aegypti) in 495 Australia. Medical and veterinary entomology. 2013 Sep;27(3):313-22.

496 22. Massad E, Amaku M, Coutinho FA, Struchiner CJ, Lopez LF, Wilder-Smith A, 497 Burattini MN. Estimating the size of Aedes aegypti populations from dengue incidence 498 data: Implications for the risk of yellow fever outbreaks. Infectious Disease Modelling. 4992017 Nov 1;2(4):441-54.

500 23. Saarman NP, Gloria $\square$ Soria A, Anderson EC, Evans BR, Pless E, Cosme LV, 501 Gonzalez $\square$ Acosta C, Kamgang B, Wesson DM, Powell JR. Effective population sizes of a 502 major vector of human diseases, Aedes aegypti. Evolutionary applications. 2017 503 Dec;10(10):1031-9.

504 24. Endersby NM, Hoffmann AA, White VL, Ritchie SA, Johnson PH, Weeks AR. 505 Changes in the genetic structure of Aedes aegypti (Diptera: Culicidae) populations in 506 Queensland, Australia, across two seasons: implications for potential mosquito releases. 507 Journal of Medical Entomology. 2011 Sep 1;48(5):999-1007.

508 25. Hale ML, Burg TM, Steeves TE. Sampling for microsatellite-based population 509 genetic studies: 25 to 30 individuals per population is enough to accurately estimate allele 510 frequencies. PloS one. 2012 Sep 12;7(9):e45170.

511 26. Philippine Statistics Authority: Population and Housing. http://psa.gov.ph/ (2016). 512 Accessed on Jun 2016

513 27. Asia Development Bank. Urban metabolism of six Asian cities: Metro Manila. 514 Mandaluyong City, Philippines: Asian Development Bank. 2014 pp. 29-34

515 28. Philippine GIS Data Clearinghouse. National Capital Region. Available online: 516 www.philgis.org (accessed on 8 November 2015)

517 29. Rueda LM. Pictorial keys for the identification of mosquitoes (Diptera: Culicidae) 518 associated with dengue virus transmission. Walter Reed Army Inst Of Research 519 Washington Dc Department Of Entomology; 2004 Aug 3.

520 30. Allendorf FW, Phelps SR. Use of allelic frequencies to describe population 521 structure. Canadian Journal of Fisheries and Aquatic Sciences. 1981 Dec 1;38(12):1507-14.

522 31. Peterman W, Brocato ER, Semlitsch RD, Eggert LS. Reducing bias in population 523 and landscape genetic inferences: the effects of sampling related individuals and multiple 524 life stages. PeerJ. 2016 Mar 14;4:e1813. 
525 32. Goldberg CS, Waits LP. Quantification and reduction of bias from sampling larvae

526 to infer population and landscape genetic structure. Molecular Ecology Resources. 2010

527 Mar;10(2):304-13.

528 33. Crane S. DNA Extraction From Archival Museum Insect Specimens. 2011.

529 Accessed Mar 2015. Available from: https://s3-eu-west-1.amazonaws.com/pfigshare-u-

530 files/1114092/extractionmuseum.pdf

531 34. Slotman MA, Kelly NB, Harrington LC, Kitthawee S, Jones JW, Scott TW,

532 Caccone A, Powell JR. Polymorphic microsatellite markers for studies of Aedes aegypti

533 (Diptera: Culicidae), the vector of dengue and yellow fever. Molecular Ecology Resources.

5342007 Jan 1;7(1):168-71.

535 35. Van Oosterhout C, Hutchinson WF, Wills DP, Shipley P. MICRO $\square$ CHECKER:

536 software for identifying and correcting genotyping errors in microsatellite data. Molecular

537 Ecology Resources. 2004 Sep 1;4(3):535-8

538 36. Rousset F. genepop'007: a complete re $\square$ implementation of the genepop software

539 for Windows and Linux. Molecular ecology resources. 2008 Jan 1;8(1):103-6.

540 37. Rousset F. GENEPOP (Version 1.2): Population genetics software for exact tests 541 and ecumenicalism. J. Hered .. 1995; 83: 239.

542 38. Kalinowski ST. Counting alleles with rarefaction: private alleles and hierarchical 543 sampling designs. Conservation genetics. 2004 Aug 1;5(4):539-43.

544 39. Kalinowski ST. hp $\square$ rare 1.0: a computer program for performing rarefaction on 545 measures of allelic richness. Molecular Ecology Resources. 2005 Mar 1;5(1):187-9.

546 40. Peakall R, Smouse PE. GenAlEx 6.5: genetic analysis in Excel. Population genetic 547 software for teaching and researchdan update. Bioinformatics 28, 2537e2539.

548 41. Excoffier L, Lischer HE. Arlequin suite ver 3.5: a new series of programs to 549 perform population genetics analyses under Linux and Windows. Molecular ecology 550 resources. 2010 May 1;10(3):564-7.

551 42. Slatkin M, Barton NH. A comparison of three indirect methods for estimating 552 average levels of gene flow. Evolution. 1989 Nov 1;43(7):1349-68.

553 43. Müllner D. fastcluster: Fast hierarchical, agglomerative clustering routines for R 554 and Python. Journal of Statistical Software. 2013 May 29;53(9):1-8.

555 44. Charrad M, Ghazzali N, Boiteau V, Niknafs A, Charrad MM. Package 'NbClust'. 556 Journal of Statistical Software. 2014 Oct 15;61:1-36. 
557 45. R Development Core Team. R: A language and environment for statistical 558 computing. R Foundation for Statistical Computing, Vienna, Austria. 2016.

559 46. Pritchard JK, Stephens M, Donnelly P (2000) Inference of population structure 560 using multilocus genotype data. Genetics 155: 945-959. pmid:10835412

561 47. Earl DA, vonHoldt BM (2012) STRUCTURE HARVESTER: a website and 562 program for visualizing STRUCTURE output and implementing the Evanno method. 563 Conservation Genetics Resources 4: 359-361.

564 48. Evanno G, Regnaut S, Goudet J. Detecting the number of clusters of individuals 565 using the software structure: a simulation study. Mol Ecol. 2005;14: 2611-2620. 566 pmid:15969739

567 49. Jakobsson M, Rosenberg NA. CLUMPP: a cluster matching and permutation 568 program for dealing with label switching and multimodality in analysis of population 569 structure. Bioinformatics. 2007 May 7;23(14):1801-6.

570 50. Rosenberg NA. DISTRUCT: a program for the graphical display of population 571 structure. Molecular Ecology Resources. 2004 Mar 1;4(1):137-8.

572 51. Vincenty T. Direct and inverse solutions of geodesics on the ellipsoid with 573 application of nested equations. Survey review. 1975 Apr 1;23(176):88-93.

574 52. Brown JE, Evans BR, Zheng W, Obas V, Barrera $\square$ Martinez L, Egizi A, Zhao H, 575 Caccone A, Powell JR. Human impacts have shaped historical and recent evolution in 576 Aedes aegypti, the dengue and yellow fever mosquito. Evolution. 2014 Feb 1;68(2):514-25.

577 53. Egizi A, Kiser J, Abadam C, Fonseca DM. The hitchhiker's guide to becoming 578 invasive: exotic mosquitoes spread across a US state by human transport not autonomous 579 flight. Molecular ecology. 2016 Jul 1;25(13):3033-47.

580 54. Fonzi E, Higa Y, Bertuso AG, Futami K, Minakawa N. Human-mediated marine 581 dispersal influences the population structure of Aedes aegypti in the Philippine Archipelago. 582 PLoS neglected tropical diseases. 2015 Jun 3;9(6):e0003829.

583 55. Chadee DD. Seasonal incidence and horizontal distribution patterns of oviposition 584 by Aedes aegypti in an urban environment in Trinidad, west Indies. Journal of the 585 American Mosquito Control Association. 1992 Sep 1;8(3):281-4.

586 56. Suleman M, Arshad M, Khan K. Yellowfever mosquito (Diptera: Culicidae) 587 introduced into Landi Kotal, Pakistan, by tire importation. Journal of medical entomology. 5881996 Jul 1;33(4):689-93.

589 57. Sukehiro N, Kida N, Umezawa M, Murakami T, Arai N, Jinnai T, Inagaki S, 590 Tsuchiya H, Maruyama H, Tsuda Y. First report on invasion of yellow fever mosquito, 
591 Aedes aegypti, at Narita International Airport, Japan in August 2012. Japanese journal of 592 infectious diseases. 2013;66(3):189-94.

593 58. Gubler DJ. Prevention and control of Aedes aegypti-borne diseases: lesson learned 594 from past successes and failures. AsPac J Mol Biol Biotechnol. 2011;19(3):111-4.

595 59. Sayson SL, Gloria-Soria A, Powell JR, Edillo FE. Seasonal genetic changes of 596 Aedes aegypti (Diptera: Culicidae) populations in selected sites of Cebu City, Philippines. 597 Journal of medical entomology. 2015 Aug 14;52(4):638-46.

598 60. Getis A, Morrison AC, Gray K, Scott TW. Characteristics of the spatial pattern of 599 the dengue vector, Aedes aegypti, in Iquitos, Peru. InPerspectives on Spatial Data Analysis 6002010 (pp. 203-225). Springer, Berlin, Heidelberg.

601 61. Reiter P, Amador MA, Anderson RA, Clark GG. Dispersal of Aedes aegypti in an 602 urban area after blood feeding as demonstrated by rubidium-marked eggs. The American 603 journal of tropical medicine and hygiene. 1995 Feb 1;52(2):177-9.

604 62. Hammond SN, Gordon AL, Lugo ED, Moreno G, Kuan GM, López MM, López JD, 605 Delgado MA, Valle SI, Espinoza PM, Harris E. Characterization of Aedes aegypti (Diptera: 606 Culcidae) production sites in urban Nicaragua. Journal of medical entomology. 2007 Sep $607 \quad 1 ; 44(5): 851-60$.

608 63. Bawalan RJ, Salazar N, Heralde F. Transovarial Transmission of Dengue Virus in 609 Aedes aegypti: A Case in Quezon City, Philippines. Acta medica Philippina 2014 Dec 610 48(4):23-29

611 64. Obra G. Development of Sterile Insect Technique for Dengue Mosquito Vector, 612 Aedes aegypti using Gamma Irradiation. Proceedings of NRCP-Symposium on Dengue 613 Researches. December 2014. The Bayleaf, Intramuros, Manila

614 65. Guedes DR, Cordeiro MT, Melo-Santos MA, Magalhaes T, Marques E, Regis L, 615 Furtado AF, Ayres CF, 2010. Patient-based dengue virus surveillance in Aedes aegypti 616 from Recife, Brazil. J Vector Borne Dis 47: 67-75.

617 66. Stoddard ST, Morrison AC, Vazquez-Prokopec GM, Paz-Soldan V, Kochel TJ, 618 Kitron U, Elder JP, Scott TW, 2009. The role of human movement in the transmission of 619 vector-borne pathogens. PLoS Negl Trop Dis 3(7): e481.

620 67. Stoddard ST, et al., 2013. House-to-house human movement drives dengue virus 621 transmission. Proc Natl Acad Sci USA 110(3): 994-999.

622 68. Zarate-Nahon EA, et al., 2013. Aedes aegypti mosquitoes at nonresidential sites 623 might be related to transmission of dengue virus in Monterrey, Northeastern Mexico. 624 Southwest Entomol 38(3): 465-476 
625 69. Hemme RR, Thomas CL, Chadee DD, Severson DW. Influence of urban landscapes

626 on population dynamics in a short-distance migrant mosquito: evidence for the dengue

627 vector Aedes aegypti. PLoS Neglected Tropical Diseases. 2010 Mar 16;4(3):e634.

628 70. Dutra HL, dos Santos LM, Caragata EP, Silva JB, Villela DA, Maciel-de-Freitas R, 629 Moreira LA. From lab to field: the influence of urban landscapes on the invasive potential 630 of Wolbachia in Brazilian Aedes aegypti mosquitoes. PLoS neglected tropical diseases. 6312015 Apr 23;9(4):e0003689.

632

633

SUPPORTING INFORMATION

634

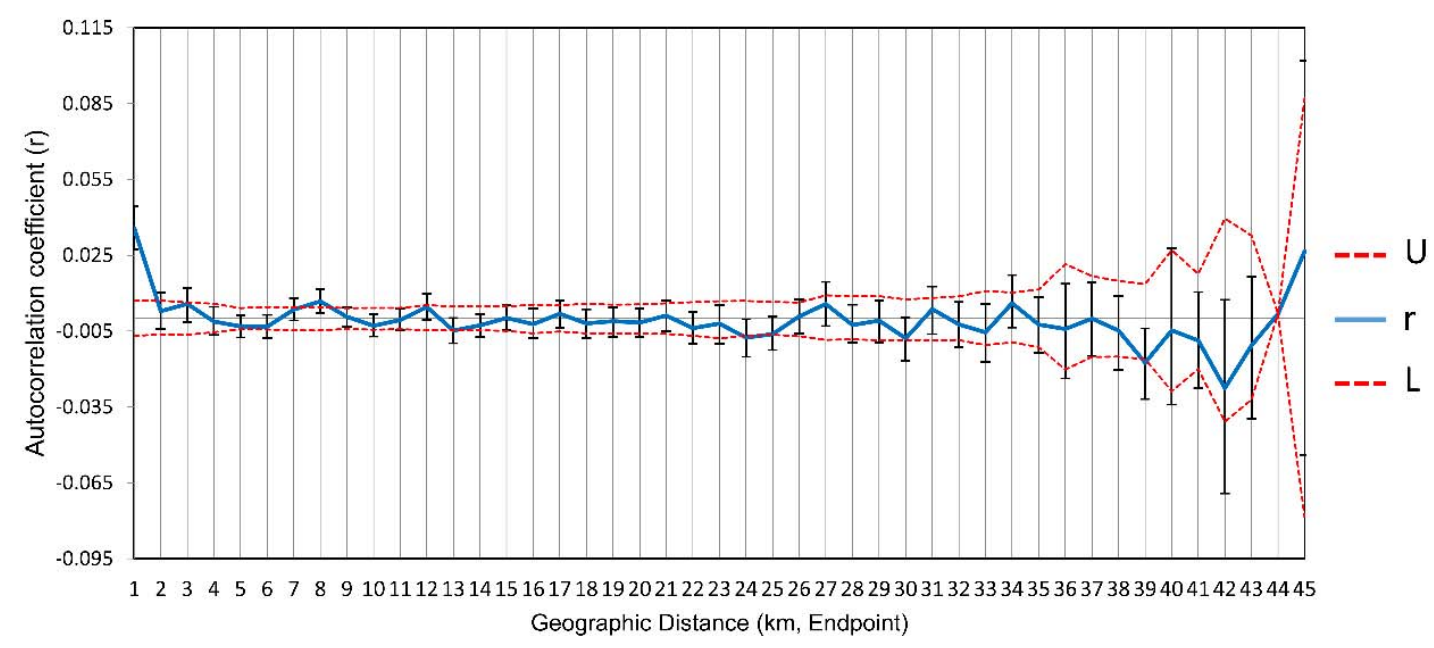

636 Figure S1. Correlogram of spatial autocorrelation showing the coefficient (r) up to $45 \mathrm{~km}$

637 with $1 \mathrm{~km}$ intervals. $\mathrm{U}$ and $\mathrm{L}$ are upper and lower confidence interval limit respectively. 


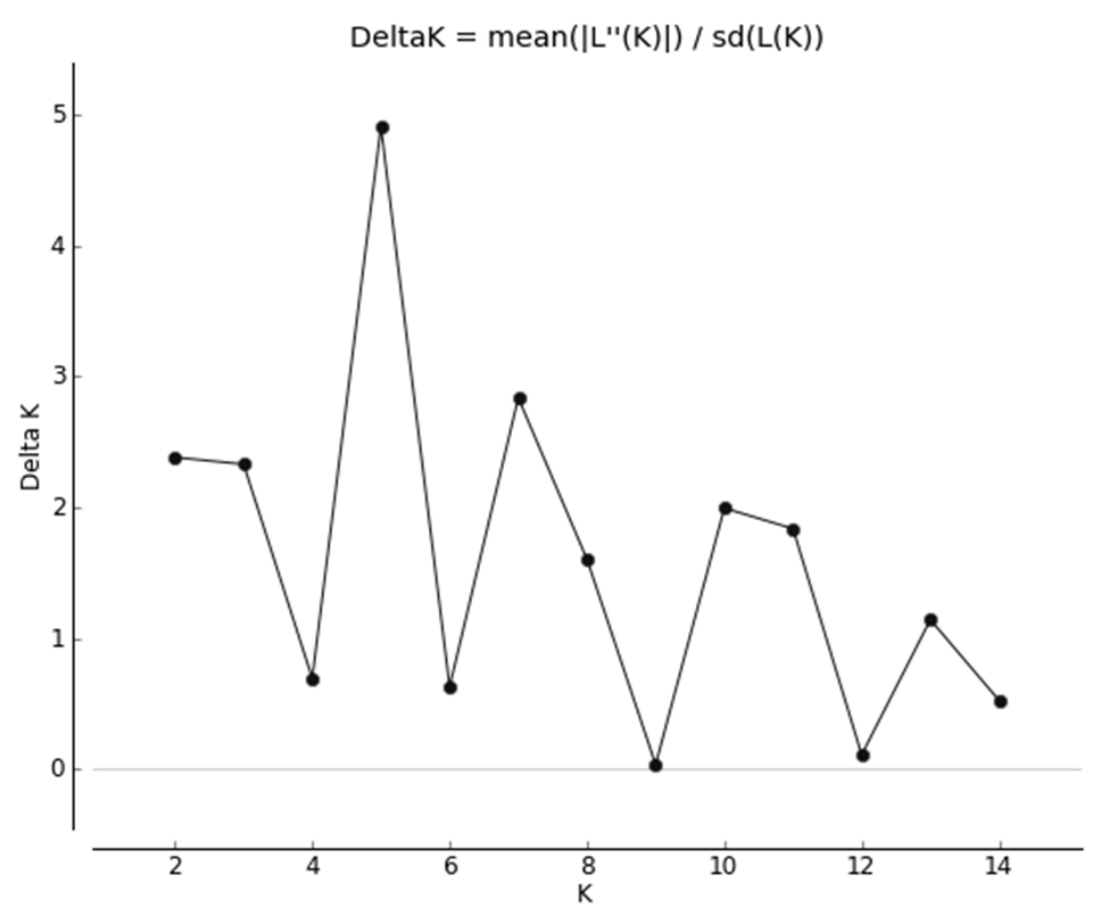

639 Figure S2. Graph of $\Delta \mathrm{K}$ against $\mathrm{K}$ showing $\mathrm{K}=5$ as the probable number of genetic 640 clusters

641 Table S1. Ae aegypti collection sites and population size in Metropolitan Manila, 642 Philippines

643 Table S2. List and Characteristics of Microsatellites markers in Ae. aegypti used in this 644 study

645 Table S3. Analysis of the Genetic Diversity of Ae aegypti using 11 microsatellite loci

646 Table S4. Null allele frequency estimates per locus per Ae aegypti populations

647 Table S5. Allele frequencies of the 11 microsatellite loci in Ae aegypti populations in 648 Metropolitan Manila

649 Table S6. Genetic differentiation using $F_{\text {ST }}$ (below diagonal) and Gene flow (Nm) (above 650 diagonal) of Ae aegypti populations in Metropolitan Manila, Philippines 